\title{
Born-Jordan and Weyl Quantizations of the 2D Anisotropic Harmonic Oscillator
}

Giovanni RASTELLI

Dipartimento di Matematica, Università di Torino, Torino, via Carlo Alberto 10, Italy

E-mail: giovanni.rastelli@unito.it

Received July 15, 2016, in final form August 15, 2016; Published online August 17, 2016

http://dx.doi.org/10.3842/SIGMA.2016.081

\begin{abstract}
We apply the Born-Jordan and Weyl quantization formulas for polynomials in canonical coordinates to the constants of motion of some examples of the superintegrable 2D anisotropic harmonic oscillator. Our aim is to study the behaviour of the algebra of the constants of motion after the different quantization procedures. In the examples considered, we have that the Weyl formula always preserves the original superintegrable structure of the system, while the Born-Jordan formula, when producing different operators than the Weyl's one, does not.
\end{abstract}

Key words: Born-Jordan quantization; Weyl quantization; superintegrable systems; extended systems

2010 Mathematics Subject Classification: 81S05; 81R12; 70H06

\section{Introduction}

Max Born and Pascual Jordan proposed a first model of quantization in [3], restricted to one-dimensional systems, a model generalized to $n$-dimensional systems, together with Werner Heisenberg, in [4]. Hermann Weyl introduced in [14, 15] a general quantization scheme based on the Fourier transform formula. The Born-Jordan and Weyl quantizations produce in general different operators from the same classical function of momenta and coordinates. The two quantization methods, however, coincide on natural Hamiltonians in $n$-dimensional Euclidean spaces. This property and many others of the two quantizations are studied in the recent book [9], that is our main source for the following discussion.

We consider here the Born-Jordan and Weyl quantizations applied to the algebra of constants of motion of the 2D anisotropic harmonic oscillator. This system admits the maximal number of three functionally independent constants of motion (and it is said to be superintegrable) whenever the ratio of the parameters is a rational number. Otherwise, the independent constants of motion are just two.

Our aim here is to check the behaviour of the Born-Jordan and Weyl quantizations of the anisotropic harmonic oscillator with respect to the integrability and superintegrability of the resulting quantum system for some particular choice of the ratio of the parameters. This aspect of the two quantization procedures is not considered in [9].

We use here the same expression for the classical 2D anisotropic harmonic oscillator and its independent constants of motion that is employed in [7]. The $\mathrm{nD}$ anisotropic harmonic oscillator is there considered as an "extended system", a particular structure of some natural Hamiltonians that allows the existence of polynomial constants of motion of higher degree, described in $[7,8]$. The interest in using such a construction here comes from other our studies in progress on the quantization of extended systems (see for example [6]).

We consider briefly also the factorization in annihilation-creation operators for the $2 \mathrm{D}$ anisotropic harmonic oscillator as given in [11], to check how the corresponding classical integrals are 
quantized by applying again Born-Jordan and Weyl procedures. The examples are confronted with those arising from the extension procedure.

We can apply here the simpler formulas for Born-Jordan and Weyl quantizations for monomials in coordinates and momenta discussed in [9].

The quantization procedures of classical quantities are not exhausted by Born-Jordan's and Weyl's approaches. Several techniques have been developed since the beginning of the quantum era, many of them specific for the particular system considered.

Indeed, there is no unique way to assign quantum operators to classical quantities in a meaningful way. Hermiticity of the operator is, usually, a necessary requirement and it is obtained by some symmetrization procedure, however not uniquely determined, see for example [13].

The problem of preserving the algebra of the constants of motion of a Hamiltonian system after quantization is object of many recent studies, see for example [1, 5, 10, 12] and references therein, for solutions in flat and non-flat manifolds.

For superintegrability and quantization in classical and quantum systems see also [12], in particular for the definition of quantum superintegrable systems, and [5].

\section{Born-Jordan and Weyl quantizations of monomials}

In $[3,4,9]$ the Born-Jordan quantization of monomials in coordinates $\left(x_{i}, p_{i}\right)$ is determined by the following general rules,

$$
\left[\hat{x}_{i}, \hat{p}_{j}\right]=i \hbar \delta_{i j}, \quad\left[\hat{x}_{i}, \hat{x}_{j}\right]=0, \quad\left[\hat{p}_{i}, \hat{p}_{j}\right]=0,
$$

where $\delta_{i j}=1$ for $i=j$ and zero otherwise, for any quantization of the coordinates $x_{i} \rightarrow \hat{x}_{i}$ and of the momenta $p_{i} \rightarrow \hat{p}_{i}$, and by

$$
x_{i}^{r} p_{i}^{s} \rightarrow \frac{1}{s+1} \sum_{k=0}^{s} \hat{p}_{i}^{s-k} \hat{x}_{i}^{r} \hat{p}_{i}^{k},
$$

for the monomials with same indices. When the indices are different, the operators commute by the general quantization rules of above and their quantization is therefore straightforward.

For the Weyl quantization [9, 14], the general rules are the same of above and, for the monomials, we have instead

$$
x_{i}^{r} p_{i}^{s} \rightarrow \frac{1}{2^{s}} \sum_{k=0}^{s}\left(\begin{array}{l}
s \\
k
\end{array}\right) \hat{p}_{i}^{s-k} \hat{x}_{i}^{r} \hat{p}_{i}^{k} .
$$

The standard realization of the operators $\hat{x}_{i}$ and $\hat{p}_{i}$, at least for Cartesian coordinates, are

$$
\hat{x}_{i} \phi=x_{i} \phi, \quad \hat{p}_{i} \phi=-i \hbar \frac{\partial}{\partial x_{i}} \phi,
$$

for any function $\phi\left(x_{j}\right)$. We employ in the following this standard quantization of the canonical coordinates.

It is easy to check that for $r=s=1$ the Born-Jordan and Weyl quantizations of monomials coincide, but differ for $r, s \geq 2$.

Many properties of the Born-Jordan quantization, generalized to any function of coordinates and momenta, and of the Weyl quantization are considered into details in [9] and the different characteristics are discussed.

We focus here our analysis on the effect of the two quantizations on the first integrals of a particular superintegrable system. We check in some examples if the quantized first integrals commute with the Hamiltonian operator, that is, if the algebraic structure of the constants of motion is preserved by the different formulas of quantization, an issue not considered in [9]. 


\section{The superintegrable 2D anisotropic harmonic oscillator}

In order to express the first integrals of the system, we can write the Hamiltonian of the superintegrable 2D anisotropic harmonic oscillator in the form of an extended Hamiltonian [7] as follows

$$
H_{m, n}=\frac{1}{2}\left(p_{u}^{2}+\left(\frac{m}{n}\right)^{2} p_{x}^{2}\right)+\omega^{2}\left(\frac{m}{n}\right)^{2}\left(x^{2}+u^{2}\right),
$$

where $(x, u)$ are coordinates in the Euclidean plane, $\omega \in \mathbb{R}$ and $m, n \in \mathbb{N} \backslash\{0\}$.

Two independent first integrals of $H_{m, n}$ are $H_{m, n}$ itself and

$$
L=\frac{1}{2} p_{x}^{2}+\omega^{2} x^{2},
$$

that is associated with the separability of the Hamilton-Jacobi equation of $H_{m, n}$ in coordinates $(u, x)$.

If we put

$$
G_{n}=\sum_{k=0}^{\left[\frac{n-1}{2}\right]}\left(\begin{array}{c}
n \\
2 k+1
\end{array}\right)\left(-2 \omega^{2}\right)^{k} x^{2 k+1} p_{x}^{n-2 k-1},
$$

where $[a]$ denotes the integer part of $a$, and $X_{L}$ is the Hamiltonian vector field of the function $L$, then, adapting to our case the more general theorem proved in [7], we have

Proposition 1. For any couple of positive integers $(m, n)$, the function $K_{m, n}$ is a first integral of $H_{m, n}$, where

$$
K_{m, n}=P_{m, n} G_{n}+D_{m, n} X_{L}\left(G_{n}\right),
$$

with

$$
\begin{aligned}
P_{m, n} & =\sum_{k=0}^{[m / 2]}\left(\begin{array}{c}
m \\
2 k
\end{array}\right)\left(-\frac{m}{n} u\right)^{2 k} p_{u}^{m-2 k}\left(-2 \omega^{2}\right)^{k}, \\
D_{m, n} & =\frac{1}{n} \sum_{k=0}^{[(m-1) / 2]}\left(\begin{array}{c}
m \\
2 k+1
\end{array}\right)\left(-\frac{m}{n} u\right)^{2 k+1} p_{u}^{m-2 k-1}\left(-2 \omega^{2}\right)^{k}, \quad m>1,
\end{aligned}
$$

and $D_{1, n}=-\frac{m}{n^{2}} u$.

We can introduce the usual Cartesian coordinates $(x, y)$ by leaving $x, p_{x}$ unchanged and putting

$$
u=\frac{n}{m} y, \quad p_{u}=\frac{m}{n} p_{y},
$$

so that

$$
H_{m, n}=\left(\frac{m}{n}\right)^{2}\left(\frac{1}{2}\left(p_{x}^{2}+p_{y}^{2}\right)+\omega^{2}\left(x^{2}+\left(\frac{n}{m}\right)^{2} y^{2}\right)\right) .
$$

In the following, we consider the $H_{m, n}$ of above by dropping the negligible overall factor $\left(\frac{m}{n}\right)^{2}$.

The classical and quantum superintegrability of the $n \mathrm{D}$ anisotropic harmonic oscillator has been studied in [11]. The quantization of the classical system is there obtained by introducing creation and annihilation operators. This technique is widely in use today (see for example [5] and [2], where the anisotropic harmonic oscillator is generalized to 2D constant-curvature 
manifolds obtaining new classical and quantum superintegrable systems) and can have some application towards the quantization of extended systems.

The Jauch-Hill Hamiltonian of the anisotropic harmonic oscillator is

$$
H_{J H}=\frac{1}{2}\left(\frac{p_{1}^{2}}{M_{1}}+\frac{p_{2}^{2}}{M_{2}}+M_{1} \omega_{1}^{2} q_{1}^{2}+M_{2} \omega_{2}^{2} q_{2}^{2}\right),
$$

and coincide with $H_{m, n}$ if we put

$$
x=\sqrt{M_{1}} q_{1}, \quad p_{1}=\sqrt{M_{1}} p_{x}, \quad y=\sqrt{M_{2}} q_{2}, \quad p_{2}=\sqrt{M_{2}} p_{y},
$$

with

$$
m \omega_{2}=n \omega_{1}, \quad \omega_{1}=\sqrt{2} \omega .
$$

The classical first integrals given in [11] become

$$
F_{1}(m, n)=\frac{1}{2}\left(b_{1}^{n} b_{2}^{* m}+b_{1}^{* n} b_{2}^{m}\right), \quad F_{2}(m, n)=-\frac{i}{2}\left(b_{1}^{n} b_{2}^{* m}-b_{1}^{* n} b_{2}^{m}\right),
$$

where

$$
b_{1}=\frac{1}{\sqrt{2 \omega_{1}}}\left(p_{x}-i \omega_{1} x\right), \quad b_{1}^{*}=\frac{1}{\sqrt{2 \omega_{1}}}\left(p_{x}+i \omega_{1} x\right),
$$

and similarly $b_{2}, b_{2}^{*}$ in function of $y, p_{y}, \omega_{2}$. The corresponding quantum operators are obtained simply by substituting $p_{x}, p_{y}$ with $\hat{p}_{x}, \hat{p}_{y}$ in the expressions of above.

The first integrals obtained with the two methods of above are different, having for example, up to constant factors,

$$
K_{1,1}=x p_{y}-y p_{x}, \quad F_{1}(1,1)=p_{x} p_{y}+\omega^{2} x y .
$$

\section{The two quantizations of the constants of motion of the oscillator}

The Born-Jordan and Weyl quantizations of both $H_{m, n}$ and $L$ clearly coincide, being in both cases

$$
\begin{aligned}
& H_{m, n} \rightarrow \hat{H}_{m, n}=-\frac{\hbar^{2}}{2}\left(\frac{\partial^{2}}{\partial x^{2}}+\frac{\partial^{2}}{\partial y^{2}}\right)+\omega^{2}\left(x^{2}+\left(\frac{n}{m}\right)^{2} y^{2}\right), \\
& L \rightarrow \hat{L}=-\frac{\hbar^{2}}{2} \frac{\partial^{2}}{\partial x^{2}}+\omega^{2} x^{2} .
\end{aligned}
$$

The operators are clearly independent and commuting, so that the integrability of the system is preserved by both the quantizations.

The Born-Jordan and Weyl quantizations of $K_{1,1}, K_{2,1}$ and $K_{3,1}$ coincide and commute with the corresponding Hamiltonian operators $\hat{H}_{m, n}$. Things become different for $(m, n)=(4,1)$. Indeed

Proposition 2. The first integral $K_{4,1}$ of $H_{4,1}$ is

$$
K_{4,1}=256\left(x p_{y}^{4}-y p_{x} p_{y}^{3}-\frac{3}{4} \omega^{2} x y^{2} p_{y}^{2}+\frac{\omega^{2}}{8} y^{3} p_{x} p_{y}+\frac{\omega^{4}}{64} x y^{4}\right) .
$$


By applying to $K_{4,1}$ the Weyl quantization formula (2), we have the Weyl operator

$$
\begin{aligned}
\hat{K}_{4,1}^{\mathrm{W}}= & 256\left(\hbar^{4}\left(x \frac{\partial^{4}}{\partial y^{4}}-y \frac{\partial^{4}}{\partial x \partial y^{3}}\right)-\frac{3 \hbar^{4}}{2} \frac{\partial^{3}}{\partial x \partial y^{2}}+\frac{\hbar^{2} \omega^{2}}{8}\left(6 x y^{2} \frac{\partial^{2}}{\partial y^{2}}-y^{3} \frac{\partial^{2}}{\partial x \partial y}\right)\right. \\
& \left.+\hbar^{2} \omega^{2} y\left(\frac{3}{2} x \frac{\partial}{\partial y}-\frac{3}{16} y \frac{\partial}{\partial x}\right)+\frac{\omega^{4}}{64} x y^{4}+\frac{3 \hbar^{2} \omega^{2}}{8} x\right),
\end{aligned}
$$

and, by applying to $K_{4,1}$ the formula (1), we get the Born-Jordan operator

$$
\hat{K}_{4,1}^{\mathrm{BJ}}=\hat{K}_{4,1}^{\mathrm{W}}+32 \hbar^{2} \omega^{2} x .
$$

The computation of the commutators gives

$$
\left[\hat{H}_{4,1}, \hat{K}_{4,1}^{\mathrm{W}}\right]=0, \quad\left[\hat{H}_{4,1}, \hat{K}_{4,1}^{\mathrm{BJ}}\right]=-32 \hbar^{4} \omega^{2} \frac{\partial}{\partial x} .
$$

Proof. We have from (4) $G_{1}=x$, therefore $X_{L} G_{1}=p_{x}$. The application of Proposition 1 to $(3)$ with $(m, n)=(4,1)$ followed by the transformation of coordinates (5) gives

$$
P_{4,1}=256 p_{y}^{4}-192 \omega^{2} y^{2} p_{y}^{2}+4 \omega^{2} y, \quad D_{4,1}=-256 y p_{y}^{3}+32 \omega^{2} y^{3} p_{y},
$$

and we get (7). We observe now that, both for Born-Jordan and Weyl quantizations, we have

$$
\hat{K}_{4,1}=\hat{P}_{4,1} \hat{x}+\hat{D}_{4,1} \hat{p}_{x}
$$

where the order of the operators is immaterial, because $P_{4,1}$ and $D_{4,1}$ depend uniquely on $\left(p_{y}, y\right)$. Therefore, we need to apply the two quantizations to $P_{4,1}$ and $D_{4,1}$ only, since the quantizations coincide on $x$ and $p_{x}$. By applying (1) and (2) to the monomials $y^{2} p_{y}^{2}, y p_{y}^{3}$ and $y^{3} p_{y}$, we have

$$
\hat{P}_{4,1}=256 \hat{p}_{y}^{4}-192 \omega^{2} \hat{Q}_{1}+4 \omega^{2} \hat{y}, \quad \hat{D}_{4,1}=-256 \hat{Q}_{2}+32 \omega^{2} \hat{Q}_{3},
$$

where

$$
\hat{Q}_{2}=i \frac{\hbar^{3}}{2}\left(2 y \frac{\partial^{3}}{\partial y^{3}}+3 \frac{\partial^{2}}{\partial y^{2}}\right), \quad \hat{Q}_{3}=-i \frac{\hbar}{2} y^{2}\left(2 y \frac{\partial}{\partial y}+3\right),
$$

coincide for both quantizations, and, for the Weyl case,

$$
\hat{Q}_{1}=-\frac{\hbar^{2}}{2}\left(2 y^{2} \frac{\partial^{2}}{\partial y^{2}}+4 y \frac{\partial}{\partial y}+1\right)
$$

while, for the Born-Jordan case,

$$
\hat{Q}_{1}=-\frac{\hbar^{2}}{3}\left(3 y^{2} \frac{\partial^{2}}{\partial y^{2}}+6 y \frac{\partial}{\partial y}+2\right) .
$$

We obtain in this way (8) and (9).

We can finally compute the commutators of $\hat{H}_{4,1}$ with $\hat{K}_{4,1}^{\mathrm{W}}$ and $\hat{K}_{4,1}^{\mathrm{BJ}}$ with the help of the formula

$$
\left[\hat{H}_{4,1}, \hat{K}_{4,1}\right]=\hat{p}_{x}\left(-i \hbar \hat{P}_{4,1}+\left[\hat{H}_{4,1}, \hat{D}_{4,1}\right]\right)+\hat{x}\left(2 \omega^{2} i \hbar \hat{D}_{4,1}+\left[\hat{H}_{4,1}, \hat{P}_{4,1}\right]\right)
$$

after making the suitable substitutions, obtaining the (10). 
We computed the quantizations for several other values of $(m, n)$, for example $(5,1),(6,1)$, $(1,4),(3,4)$, such that $\hat{K}_{m, n}^{\mathrm{BJ}} \neq \hat{K}_{m, n}^{\mathrm{W}}$, obtaining always commutation with $\hat{H}_{m, n}$ for $\hat{K}_{m, n}^{\mathrm{W}}$ and no commutation with $\hat{H}_{m, n}$ for $\hat{K}_{m, n}^{\mathrm{BJ}}$. In these last cases, it can be observed that both $\omega$ and $\hbar$ always appear as factors in the commutator, meaning that for $\omega=0$ and for $\hbar \rightarrow 0$ the operators commute.

Analogous results are obtained from the quantizations of the first integrals $F_{1}(m, n)$ or $F_{2}(m, n)$ given in $(6)$ for several values of $(m, n)$ : the Weyl formula produces symmetry operators of the Hamiltonian, the Born-Jordan one, when giving different operators, does not.

Actually, one can conjecture that the Weyl quantizations of $K_{m, n}$ and $F_{i}(m, n)$ always commute with the Hamiltonian operator $\hat{H}_{m, n}$.

It can be observed, as one of the referees of this article pointed out, that "for the JauchHill approach the quantization using creation and annihilation operators shows very easily that the quantum extensions of $F_{1}$ and $F_{2}$ are still integrals. It is less obvious to prove that this quantization is nothing but Weyl's one".

Remark 3. The failure of the Born-Jordan quantization formula in reproducing the algebra of constants of motion at the quantum level is, actually, restricted to the particular set of generators of the algebra that we choose. We do not know in general if another choice of independent first integrals can lead to different results.

Remark 4. The failure in reproducing the algebra of the constants of motion after quantization appears also in the case of natural Hamiltonian systems on curved manifolds. In these cases, quantum corrections of the Hamiltonian operator are necessary in order to preserve the integrable or superintegrable algebraic structure $[1,10]$.

\section{Conclusions}

From the examples computed, it appears that the Born-Jordan quantization formula fails in preserving the high-degree constants of motion of the 2D anisotropic harmonic oscillator, and therefore its superintegrability, to the quantum level, differently from the Weyl formula. A study of this problem in full generality is then desirable.

\section{Acknowledgements}

I am grateful to the referees of this article for their comments and suggestions.

\section{References}

[1] Ballesteros Á., Enciso A., Herranz F.J., Ragnisco O., Riglioni D., Quantum mechanics on spaces of nonconstant curvature: the oscillator problem and superintegrability, Ann. Physics 326 (2011), 2053-2073, arXiv:1102.5494.

[2] Ballesteros Á., Herranz F.J., Kuru Ş., Negro J., The anisotropic oscillator on curved spaces: a new exactly solvable model, Ann. Physics 373 (2016), 399-423, arXiv:1605.02384.

[3] Born M., Jordan P., Zur Quantenmechanik, Z. Phys. 34 (1925), 858-888.

[4] Born M., Heisenberg W., Jordan P., Zur Quantenmechanik. II, Z. Phys. 35 (1925), 557-615.

[5] Celeghini E., Kuru Ş., Negro J., del Olmo M.A., A unified approach to quantum and classical TTW systems based on factorizations, Ann. Physics 332 (2013), 27-37.

[6] Chanu C., Degiovanni L., Rastelli G., Generalizations of a method for constructing first integrals of a class of natural Hamiltonians and some remarks about quantization, J. Phys. Conf. Ser. 343 (2012), 012101, 15 pages, arXiv:1111.0030. 
[7] Chanu C.M., Degiovanni L., Rastelli G., Extensions of Hamiltonian systems dependent on a rational parameter, J. Math. Phys. 55 (2014), 122703, 11 pages, arXiv:1310.5690.

[8] Chanu C.M., Degiovanni L., Rastelli G., The Tremblay-Turbiner-Winternitz system as extended Hamiltonian, J. Math. Phys. 55 (2014), 122701, 8 pages, arXiv:1404.4825.

[9] de Gosson M.A., Born-Jordan quantization. Theory and applications, Fundamental Theories of Physics, Vol. 182, Springer, Cham, 2016.

[10] Duval C., Valent G., Quantum integrability of quadratic Killing tensors, J. Math. Phys. 46 (2005), 053516, 22 pages, math-ph/0412059.

[11] Jauch J.M., Hill E.L., On the problem of degeneracy in quantum mechanics, Phys. Rev. 57 (1940), 641-645.

[12] Miller Jr. W., Post S., Winternitz P., Classical and quantum superintegrability with applications, J. Phys. A: Math. Theor. 46 (2013), 423001, 97 pages, arXiv:1309.2694.

[13] Post S., Winternitz P., General Nth order integrals of motion in the Euclidean plane, J. Phys. A: Math. Theor. 48 (2015), 405201, 24 pages, arXiv:1501.00471.

[14] Weyl H., Quantenmechanik und Gruppentheorie, Z. Phys. 46 (1927), 1-46.

[15] Weyl H., The theory of groups and quantum mechanics, Dover, New York, 1950. 